\title{
Comparison of body composition assessments by bioelectrical impedance and by anthropometry in premenopausal Chinese women
}

\author{
By FROUWKJE G. DE WAART ${ }^{1}$, RUOWEI LI' ${ }^{2}$ AND PAUL DEURENBERG ${ }^{1}$ \\ ${ }^{1}$ Department of Human Nutrition, Wageningen Agricultural University, PO Box 8129, 6700 EV \\ Wageningen, The Netherlands, "Institute of Nutrition and Food Hygiene, Chinese Academy of \\ Preventive Medicine, Beijing, China
}

(Received 4 March 1992 - Accepted 16 July 1992)

\begin{abstract}
Fat-free mass (FFM) was estimated in forty-seven premenopausal Chinese women, aged 18-43 years, from anthropometric data (skinfolds, body mass index (weight/height ${ }^{2}$; BMI)) or bioelectrical impedance, using several prediction formulas for body composition from the literature, and values compared with the mean of these three individual methods used as a frame of reference. In thirty-six women these values could be compared with FFM calculated from total body water (TBW) determined by $\mathrm{D}_{2} \mathrm{O}$ dilution. The prediction formulas used were developed from studies on Caucasian adults and their validity will have to be shown in populations with different ethnic backgrounds. The mean difference between FFM predicted from BMI and the frame of reference was $0.1 \mathrm{~kg}(95 \%$ confidence interval (CI) $-0.1,0.4)$, from bioelectrical impedance it was $0.5 \mathrm{~kg}(95 \% \mathrm{CI} 0.3,0.7)$, and from skinfolds it was $-0.6 \mathrm{~kg}(95 \% \mathrm{CI}-0.9,-0.4)$. The mean difference between FFM calculated from TBW and the frame of reference was higher $(2 \cdot 2 \mathrm{~kg}, 95 \% \mathrm{CI} \mathrm{1.2,3} \cdot 3)$. The results of the present study indicate that the three methods may be valid for predicting body composition in adult Chinese females, but further research is needed on development and cross-validation of prediction equations for body composition for Chinese.
\end{abstract}

Body composition: Anthropometry: Bioelectrical impedance

Many methods are available for the assessment of body composition, but only a few, e.g. anthropometry and bioelectrical impedance, are useful in field studies (Forbes, 1987; Lukaski, 1987). The advantages of these methods are that they are relatively inexpensive, non-invasive, and portable. Furthermore, bioelectrical impedance does not require highly skilled technicians. However, it should be mentioned that recently the advantage of using bioelectrical impedance above simple weight-height indices for assessing body composition has been questioned (Diaz et al. 1989; Deurenberg et al. 1991). It is of great importance that simple and cheap methods are validated for different populations.

Many equations have been developed for predicting body composition using bioelectrical impedance (Lukaski et al. 1985; Pasco \& Rutishauer, 1985; Segal et al. 1988) or anthropometric indices such as skinfold measurements (Durnin \& Womersley, 1974; Jackson \& Pollock, 1978; Jackson et al. 1980) or body mass index (weight/height ${ }^{2}$; BMI) (Womersley \& Durnin, 1977; Deurenberg et al. 1991).

These equations were all developed from studies on adult Caucasian populations and may, therefore, only be valid for this particular group. Only a few studies have been reported where the applicability of these prediction formulas was validated in black populations (Zillikens \& Conway, 1990, 1991).

\footnotetext{
Address for correspondence: Dr Paul Deurenberg, Department of Human Nutrition, Agricultural University Wageningen, PO Box 8129,6700 EV Wageningen, The Netherlands
} 
In a study on Chinese subjects by Jiang et al. (1991) body composition was studied using a multiple-tracer dilution method measuring total body water, extracellular water, plasma volume, and erythrocyte volume. Healthy Chinese male and female subjects were compared with a group of healthy Americans previously evaluated by similar dilution techniques. Jiang et al. (1991) concluded that Chinese have comparable body composition but no additional fat burden. The study by Jiang et al. (1991) was conducted on a relatively small number of subjects and the multiple-tracer techniques used are expensive and laborious, although they are highly accurate.

To our knowledge, no studies have been done on the validity of using Western prediction formulas for assessing body composition by anthropometry and bioelectrical impedance in Chinese people.

In the present study fat-free mass (FFM; $\mathrm{kg}$ ) estimated by several prediction formulas from the literature using anthropometric values (skinfolds, BMI) or bioelectrical impedance measurements was compared in forty-seven premenopausal Chinese women. In thirty-six women these values could be compared with the FFM calculated from total body water (TBW) determined by $\mathrm{D}_{2} \mathrm{O}$ dilution.

\section{SUBJECTS AND METHODS}

\section{Subjects}

Forty-seven non-pregnant premenopausal women aged 18-43 years, living in Beijing, China, were selected for this study. In thirty-six women TBW was determined by $\mathrm{D}_{2} \mathrm{O}$ dilution and FFM was calculated from TBW by dividing TBW by 0.725 (Pace \& Rathbun, 1945). All subjects had participated in a study on energy metabolism in relation to $\mathrm{Fe}$ deficiency. The subjects were involved in relatively heavy work in a cotton factory. They had no abnormalities in alanine aminotransferase (EC 2.6.1.2), chest X-ray, folic acid concentration in erythrocytes and serum, stool occult bleeding, or numbers of hookworm eggs in the stools. The subjects came to the hospital the night before the measurements were performed and stayed overnight. Each subject completed all tests in the same morning after voiding and after an overnight fast.

\section{Methods}

Anthropometry. Height and body weight were measured to the nearest $0.001 \mathrm{~m}$ and $0.1 \mathrm{~kg}$ respectively with a beam weighing scale and measuring system (SECA 220; Lameris, Utrecht, The Netherlands). The weight of the subject's clothes was measured separately and subtracted from the total weight. Percentage body fat was calculated from BMI using the age- and sex-specific prediction formula (Deurenberg et al. 1991). From percentage body fat the FFM was calculated.

Skinfold thickness was measured to the nearest $0.2 \mathrm{~mm}$ in triplicate at the triceps, biceps, subscapular and suprailiac on the right side of the body using a Holtain skinfold calliper (Holtain Ltd, Crymych, Dyfed, UK). The average of the three consecutive measurements was used in further analysis. One observer (F.D.W.) performed most of the measurements but a small number $(<10 \%)$ were performed by a second observer who had been trained by the same person to use similar techniques.

Body density $(\mathrm{kg} / \mathrm{l})$ was predicted from the logarithm of the sum of the four skinfold thickness measurements using the equations of Durnin \& Womersley (1974). Percentage body fat was calculated from the body density values obtained using Siri's (1961) equation:

$$
\text { percentage body fat }=(495 / \text { body density })-450 \text {. }
$$

FFM was calculated from percentage body fat. 
Bioelectrical impedance. All bioelectrical impedance measurements were performed with a bioelectrical impedance analyser (BIA 101; RJL Systems, Detroit, MI, USA) following the procedures described by Lukaski et al. (1985). The measurements were performed in duplicate without removing the electrodes and the mean value was used in further analyses. Measurements were taken on the right side of the body. FFM was calculated using the generalized regression equation of Segal et al. (1988), which includes resistance, height, weight, age and sex.

$D_{2} O$ dilution. Thirty-six subjects received orally an accurately weighed $10 \mathrm{~g}$ dose of $\mathrm{D}_{2} \mathrm{O}$ $\left(999 \mathrm{~g} / \mathrm{kg}\right.$ ) (Isotec, Miamisburg, OH, USA). Before administration of $\mathrm{D}_{2} \mathrm{O}$, urine was collected and a portion of the sample (blank) was retained. After administration of $\mathrm{D}_{2} \mathrm{O}$ the subjects had breakfast consisting of two slices of bread, an egg, some jam, and $250 \mathrm{ml}$ soyabean milk. Urine was collected $2.5 \mathrm{~h}$ after administration of $\mathrm{D}_{2} \mathrm{O}$ and a portion of the sample was retained. All urine samples were stored at $-20^{\circ} . \mathrm{D}_{2} \mathrm{O}$ analyses were performed by infrared absorption (Lukaski \& Johnson, 1985).

TBW was calculated from the difference in concentration of $\mathrm{D}_{2} \mathrm{O}$ in the urine before and after the dose $\left(\Delta D_{2} O\right)$, and the amount of $D_{2} O$ given:

$$
\mathrm{TBW}=\left(\text { given dose } \mathrm{D}_{2} \mathrm{O}\right) / \Delta \mathrm{D}_{2} \mathrm{O} \times 0.95 \text {. }
$$

A $5 \%$ correction for non-aqueous dilution of $\mathrm{D}_{2} \mathrm{O}$ was made. FFM was calculated from TBW with the assumption that FFM is hydrated to the extent of $720 \mathrm{~g} / \mathrm{kg}$ (Pace \& Rathbun, 1945).

\section{Statistical analysis}

The data were analysed using the Statistical Package for Social Sciences/PC (1988) programme. The estimates of FFM $(\mathrm{kg})$ by skinfolds, bioelectrical impedance and BMI were compared with the individual mean of these three methods, which was used as a frame of reference, according to the method described by Bland \& Altman (1986). Because of the lack of a 'gold' standard, it is assumed that the most valid estimate of FFM may be the mean of the three methods. Values are expressed as means and standard deviations. Differences in variables were tested using Student's $t$ test; $P<0.05$ was regarded as significant.

\section{RESULTS}

Table 1 shows some characteristics of the subjects. BMI was 21.4 (SD $4 \cdot 2$; range $17 \cdot 6$ $29 \cdot 5) \mathrm{kg} / \mathrm{m}^{2}$.

In Table 2, values for FFM ( $\mathrm{kg}$ ) calculated using different prediction formulas are given. FFM from skinfold thickness was slightly lower than those of FFM from bioelectrical impedance (Segal-prediction) and BMI. The FFM estimated by bioelectrical impedance was $40 \cdot 3$ (SD 3.7) kg. The FFM of the thirty-six women calculated from the TBW measured by $\mathrm{D}_{2} \mathrm{O}$ dilution was $42 \cdot 1$ ( $\mathrm{SD} 4 \cdot 6$ ) $\mathrm{kg}$.

Table 3 shows the mean differences between FFM predicted using a single method and the frame of reference, i.e. the mean of the three individual methods (BMI, bioelectrical impedance and skinfolds). The differences were small, although statistically significant for the FFM predicted by skinfolds and bioelectrical impedance. The mean difference between FFM calculated from $\mathrm{D}_{2} \mathrm{O}$ dilution ( $n$ 36) and the frame of reference (in these thirty-six women) was somewhat higher $(2 \cdot 2$ (SD $3 \cdot 2) \mathrm{kg}(95 \%$ confidence intervals (Cl) $1 \cdot 2,3 \cdot 3)$; $P<0.001$ ).

Fig. 1 shows the plot of the difference between predicted FFM and mean of the three individual methods $v$. the frame of reference. The differences were not related to the mean FFM. 
Table 1. Physical characteristics of the subjects

(Mean values and standard deviations)

\begin{tabular}{lcccc}
\hline & & & \\
& Mean & & SD & Range \\
\hline Age (years) & & & \\
Height (m) & $29 \cdot 3$ & $6 \cdot 2$ & $18-43$ \\
Wt (kg) & $1 \cdot 61$ & $0 \cdot 05$ & $1 \cdot 51-1 \cdot 72$ \\
Body mass index (wt/height $\left.{ }^{2} ; \mathrm{kg} / \mathrm{m}^{2}\right)$ & $55 \cdot 4$ & $7 \cdot 1$ & $42 \cdot 2-74 \cdot 9$ \\
Sum of four skinfolds $(\mathrm{mm})^{*}$ & $21 \cdot 4$ & $2 \cdot 8$ & $17 \cdot 6-29 \cdot 5$ \\
Body impedance $(\Omega)$ & $55 \cdot 7$ & $18 \cdot 9$ & $24 \cdot 9-97 \cdot 7$ \\
& 621 & 56 & $516-775$ \\
\hline
\end{tabular}

* Triceps, biceps, subscapular and supra-iliac.

Table 2. Calculated fat-free mass $(F F M)(\mathrm{kg})$ of premenopausal Chinese women using different prediction formulas

(Mean values and standard deviations)

\begin{tabular}{|c|c|c|c|}
\hline $\begin{array}{l}\text { Prediction formula for } \\
\text { FFM derived from: }\end{array}$ & Source & Mean & $\mathrm{SD}$ \\
\hline Body mass index & Deurenberg et al. (1991) & $40 \cdot 2$ & 3.3 \\
\hline Bioimpedance & Segal et al. (1988) & $40 \cdot 5$ & $3 \cdot 1$ \\
\hline Skinfolds & Durnin \& Womersley (1974) & $39 \cdot 4$ & $3 \cdot 3$ \\
\hline
\end{tabular}

For details of subjects and procedures, see Table 1 and pp. 658-659.

Table 3. Mean difference* between predicted fat-free mass (FFM) and the mean of the three individual methods used for premenopausal Chinese women $\dagger$

(Mean values and standard deviations)

\begin{tabular}{llrrr}
\hline $\begin{array}{l}\text { Predicted FFM } \\
\text { derived from: }\end{array}$ & Source & Mean & SD & $95 \% \mathrm{CI}$ \\
\hline Body mass index & Deurenberg et al. (1991) & $0 \cdot 1$ & $0 \cdot 8$ & $-0 \cdot 1-0 \cdot 4$ \\
Bioimpedance & Segal et al. (1988) & $0 \cdot 5$ & $0 \cdot 7$ & $0 \cdot 3-0 \cdot 7$ \\
Skinfolds & Durnin \& Womersley (1974) & -0.6 & $0 \cdot 8$ & $-0 \cdot 9-0 \cdot 4$ \\
\hline
\end{tabular}

CI, confidence intervals.

* Difference $=$ predicted minus the mean of the three individual methods.

$\dagger$ For details of subjects and procedures, see Table 1 and pp. 658-659.

In Fig. 2 the relationship between the frame of reference and the FFM calculated from TBW, determined by $\mathrm{D}_{2} \mathrm{O}$ dilution, is given. The standard error of the estimate $(3 \cdot 3 \mathrm{~kg})$ of this relationship was relatively large.

\section{DISCUSSION}

The prediction equations used in the present study were developed in Caucasian adults with hydrodensitometry as the reference method. They are based on a two-compartment model (body weight $=$ fat mass + FFM), and may contain errors related to deviations in the composition of the FFM from the fixed values that are assumed (Coward et al. 1988). These 

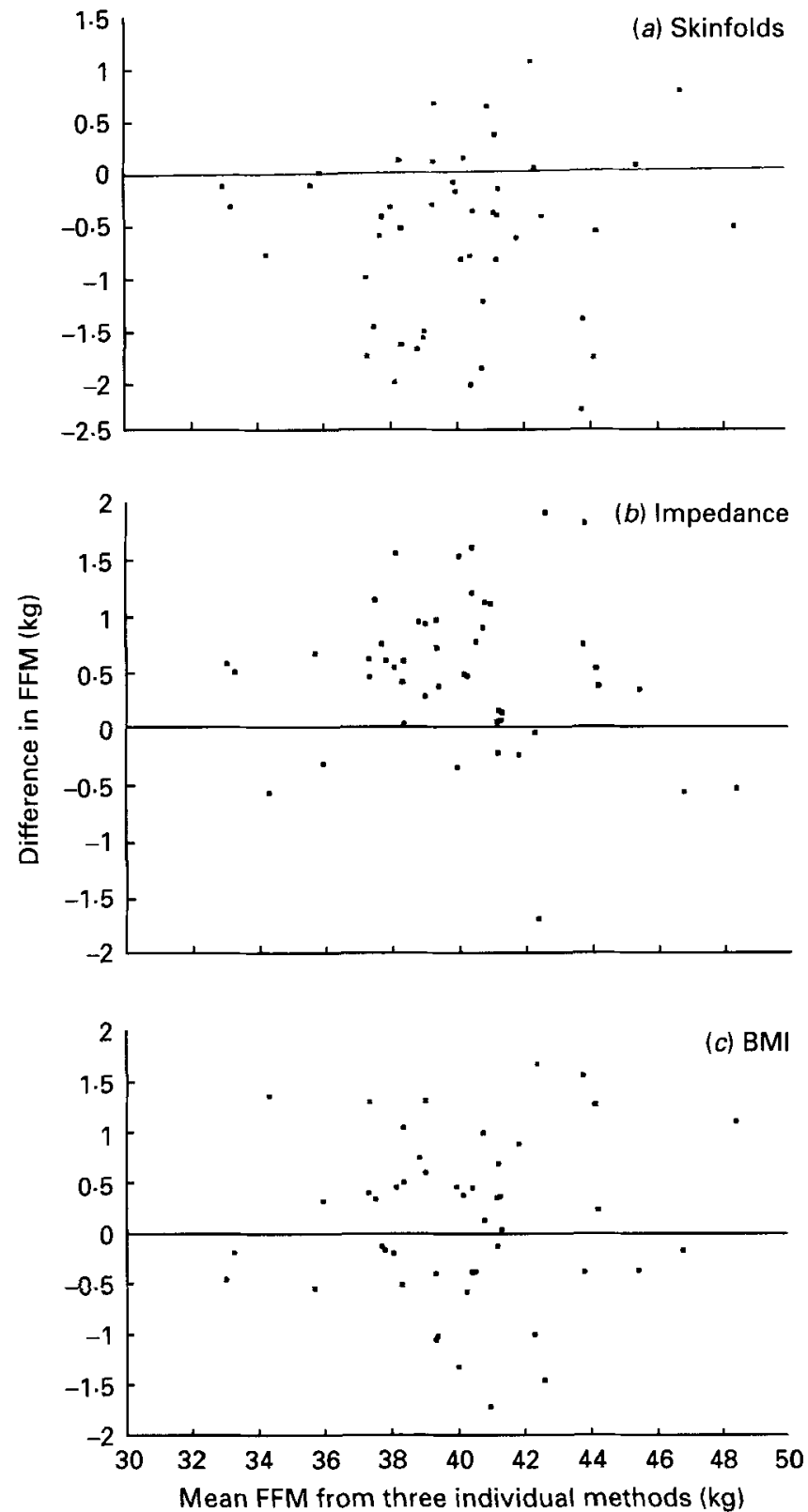

Fig. 1. Individual differences between predicted fat-free mass (FFM) from bioelectrical impedance (Segal et al. (1988), skinfold thickness (Durnin \& Womersley, 1974), body mass index (BMI) (Deurenberg et al. 1991) and the frame of reference (mean of the three individual methods) for premenopausal Chinese women. For details of subjects and procedures, see Table 1 and pp. 658-659.

assumptions may not be valid in particular for: children; elderly people, who may have serious bone demineralization (Deurenberg et al. 1989); subjects with disturbed water homeostasis; ethnic groups (Harsha et al. 1978; Shutte, 1980; Davies et al. 1988; Zillikens \& Conway, 1990, 1991; Baumgartner et al. 1991; Deurenberg et al. 1992). Baumgartner et al. (1991) suggested that loss in accuracy when equations developed in one sample are 


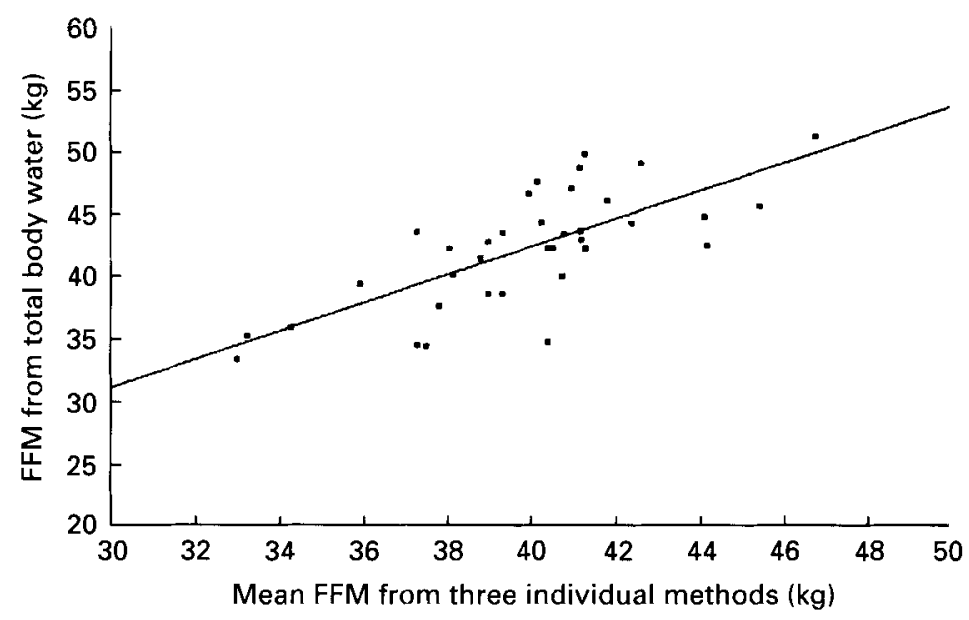

Fig. 2 Regression between fat-free mass (FFM) estimated by deuterium oxide dilution and the frame of reference (mean from the individual methods; skinfolds, bioelectrical impedance, body mass index) for premenopausal Chinese women. For details of subjects and procedures, see Table 1 and pp. 658-659. $Y=1 \cdot 1135 X-2 \cdot 28, r 0 \cdot 74$, SE of estimate $3 \cdot 3 \mathrm{~kg}$.

applied to another may be due to differences between the samples in the distributions of components of the FFM that are not accounted for when criterion estimates are obtained using two-compartment models. They suggested that prediction equations for body composition for groups in which the assumptions are doubtful should be calibrated against estimates from multi-compartment models that consider variability in FFM composition. The most important constituents to consider in this respect are water, because it is the largest component, and mineral, because it is the most dense.

Zillikens \& Conway (1990) have investigated the accuracy of generalized skinfold equations in black subjects and compared the fat pattern of black and white adults. $\mathrm{D}_{2} \mathrm{O}$ dilution was used as the reference method. They demonstrated differences in fat pattern and fat distribution between black and white adults, with black adults showing more upperbody fat deposition marked by lower values for supra-iliac: subscapular skinfold thickness compared with white adults. Despite these differences in fat distribution between black and white adults, Zillikens \& Conway (1990) found no reason to develop race-specific skinfold prediction equations. The Durnin \& Womersley (1974) equations predicted percentage body fat, estimated by $\mathrm{D}_{2} \mathrm{O}$ dilution, accurately. In another study Zillikens \& Conway (1991) compared various bioelectrical impedance prediction equations and showed that TBW was significantly underestimated in black subjects by using equations developed in white populations.

Until now no systematic body composition studies in Chinese people have been performed to check the applicability of the commonly used prediction formulas for determining body composition by anthropometry or bioelectrical impedance and, eventually, to develop new, population-specific prediction formulas.

In the present study prediction formulas developed in Caucasian adults for estimating body composition from skinfold thickness, BMI and bioelectrical impedance were compared with a frame of reference (the mean of three individual prediction formulas). For skinfold measurements the age- and sex-specific equations for Durnin \& Womersley (1974) were used; for bioelectrical impedance the prediction formula from Segal et al. (1988) was chosen because it had been cross-validated in four laboratories and a large study group was used ( $n$ 1567, of which 498 were women). The advantage of the relatively expensive bio- 
electrical impedance method over simple anthropometric measures such as weight, height and/or skinfolds has been questioned (Diaz et al. 1989; Deurenberg et al. 1991). Therefore, the sex- and age-specific prediction formula for body composition from BMI (Deurenberg et al. 1991) was also included in the analysis. Estimates of body composition from skinfold thickness, bioelectrical impedance, and BMI are based on different principles, so each estimate is independent of those produced by one of the other methods. Despite these differences in principles these methods produced estimates of FFM which differed only slightly (Tables 2 and 3, Fig. 1). In a subgroup of thirty-six women TBW was determined by $\mathrm{D}_{2} \mathrm{O}$ dilution and FFM was calculated from TBW. Because of the possible unreliability of some samples, caused by a transportation problem with weighed $\mathrm{D}_{2} \mathrm{O}$ dose and urine samples, this method was not used as the reference method. The mean FFM calculated from $\mathrm{D}_{2} \mathrm{O}$ dilution for the thirty-six women was $42 \cdot 1$ (SD 4.6 ) $\mathrm{kg}$. The mean FFM from the frame of reference in this subgroup was 39.9 (SD 3.2) $\mathrm{kg}$. The difference of $2 \cdot 2$ (SD $3 \cdot 2$ ) $\mathrm{kg}$, $(P<0.001)$ may be due to errors in the prediction formulas as well as errors in the dilution technique caused by differences in hydration and/or bone mass density for Chinese women compared with Caucasian women.

Information on body composition and methods to predict body composition in Chinese subjects is very scarce. There is a need for validation of non-invasive methods that can be used in epidemiological and clinical studies on populations other than Western societies. The results of the present study indicate that the three methods used to predict body composition are comparable and reliable for the estimation of body composition in female Chinese adults. Further research is needed, however, on the development and crossvalidation of prediction equations for Chinese subjects.

The authors thank the subjects for their participation in the project. The study was supported by the Nestlé Foundation, Switzerland.

\section{REFERENCES}

Baumgartner, R. N., Heymsfield, S. B., Lichtman, S., Wang, J. \& Pierson, R. N. (1991). Body composition in elderly people: effect of criterion estimates on predictive equations. American Journal of Clinical Nutrition $\mathbf{5 3}$, $1345-1353$

Bland, J. M. \& Altman, D. G. (1986). Statistical methods for assessing agreement between two methods of clinical measurement. Lancet i, 307-310.

Coward, W. A., Parkinson, S. A. \& Murgatroyd, P. R. (1988). Body composition measurements for nutrition research. Nutrition Research Reviews 1, 115-124.

Davies, P. S. W., Preece, M. A., Hicks, C. J. \& Halliday, D. (1988). The prediction of TBW using BI in children and adolescents. Annals of Human Biology 40, 237-240.

Deurenberg, P., Saris, W. H. M., Voorrips, L. E. \& VanStaveren, W. A. (1993). The assessment of the body composition in the elderly. Age \& Nutrition, (In the press).

Deurenberg, P., Weststrate, J. A. \& Seidell, J. C. (1991). Body mass index as a measure of body fatness: age- and sex-specific prediction formulas. British Journal of Nutrition 65, 105-114.

Deurenberg, P., Weststrate, J. A. \& van der Kooy, K. (1989). Is an adaptation of Siri's fomula for the calculation of body fat percentage from body density in the elderly necessary? European Journal of Clinical Nutrition $\mathbf{4 3}$, $559-568$.

Diaz, E. O., Villar, J., Immink, M. \& Gonzales, T. (1989). Bioimpedance or anthropometry? European Journal of Clinical Nutrition 43, 129-137.

Durnin, J. V. G. A. \& Womersley, J. (1974). Body fat assessed from total body density and its estimation from skinfold thickness: measurements on 481 men and women aged from 16 to 72 years. British Journal of Nutrition 32, 77-97.

Forbes, G. G. (1987). Human Body Composition. New York: Springer Verlag.

Harsha, D. W., Frerichs, R. R. \& Berenson, G. S. (1978). Densitometry and anthropometry of black and white children. Human Biology 50, 261-280.

Jackson, A. S. \& Pollock, M. L. (1978). Generalized equations for predicting body density of men. British Journal of Nutrition 40,497-504.

Jackson, A. S., Pollock, M. L. \& Ward, A. (1980). Generalized equations for predicting body density of women. Medicine and Science in Sports and Exercise 12, 175-182. 
Jiang, Z. M., Yang, N. F., Chou, C., Liu, Z., Sun, T., Chen, Y., Xue, B., Fei, L., Tseng, H., Browns, E., Scheltinga, M. \& Wilmore, D. W. (1991). Body composition in Chinese subjects: comparison with data from North America. World Journal of Surgery 15, 95-102.

Lukaski, H. C. (1987). Methods for the assessment of human body composition: traditional and new. American Journal of Clinical Nutrition 46, 537-556.

Lukaski, H. C. \& Johnson, P. E. (1985) A simple, inexpensive method of determining total body water using a tracer dose of $\mathrm{D}_{2} \mathrm{O}$ and infrared absorption of biological fluids. American Journal of Clinical Nutrition 41, 363-370.

Lukaski, H. C., Johnson, P. E., Bolonchuck, W. W. \& Lykken, G. I. (1985). Assessment of fat-free mass using BIM of the human body. American Journal of Clinical Nutrition 41, 810-817.

Pace, N. \& Rathbun, E. N. (1945). Studies on body composition. III. The body water and chemically combined nitrogen content in relation to fat content. Journal of Biological Chemistry 158, 685-691.

Pasco, J. A. \& Rutishauer, I. H. E. (1985). Body fat estimated from anthropometric and electrical impedance measurements. Human Nutrition: Clinical Nutrition 39, 365-369.

Shutte, J. E. (1980). Prediction of total body water in adolescent males. Human Biology 52, 381-391.

Segal, K. R., VanLoan, M., Fitzgerald, P. I., Hodgson, J. A. \& Van Itallie, T. B. (1988). Lean body mass estimation by bio-electrical impedance analysis: a four-site cross-validation study. American Journal of Clinical Nutrition 47, 7-14.

Siri, W. E. (1961). Body composition from fluid spaces and density, analysis of methods. In Techniques for Measuring Body Composition, pp. 223-244 [J. Brozek and A. Henschel, editors]. Washington, DC: National Academy of Sciences.

Statistical Package for Social Sciences/PC. (1988). Base Manual+V2.0. Chicago, Ill: SPSS Inc.

Womersley, J. \& Durnin, J. V. G. A. (1977). A comparison of the skinfold method with extent of 'overweight ' and various weight-height relationships in the assessment of obesity. British Journal of Nutrition 38, $271-284$.

Zillikens, M. C. \& Conway, J. M. (1990). Anthropometry in blacks: applicability of generalized skinfold equations and differences in fat patterning between blacks and whites. American Journal of Clinical Nutrition 52, 45-51.

Zillikens, M. C. \& Conway, J. M. (1991). The estimation of total body water by bioelectrical impedance analysis in blacks. American Journal of Human Biology 3, 25-32. 